\title{
Förster Resonance Energy Transfer Mediated Photoluminescence Quenching in Stoichiometrically Assembled CdSe/ZnS Quantum Dot-Peptide Labeled Black Hole Quencher Conjugates for Matrix Metalloproteinase-2 Sensing
}

\author{
Sreenadh Sasidharan Pillai, $* 1, * 2 \dagger$ Hiroshi Yukawa, $* 1, * 2$ Daisuke Onoshima, $* 3$ \\ Vasudevanpillai BIJU, $* 4, * 5$ and Yoshinobu BABA $* 1, * 2, * 3, * 4$ \\ *1 Graduate School of Engineering, Nagoya University, Furo-cho, Chikusa, Nagoya 464-8603, Japan \\ *2 ImPACT Research Center for Innovative Nanobiodevices, Nagoya University, Furo-cho, Chikusa, \\ Nagoya 464-8603, Japan \\ *3 Institute of Innovation for Future Society, Nagoya University, Furo-cho, Chikusa, Nagoya 464-8603, Japan \\ *4 Health Research Institute, National Institute of Advanced Industrial Science and Technology (AIST), \\ 2217-14 Hayashi-cho, Takamatsu, Kagawa 761-0395, Japan \\ *5 Research Institute for Electronic Science, Hokkaido University, Kita 20 Nishi 10, Kita, Sapporo, \\ Hokkaido 001-0020, Japan
}

\begin{abstract}
The steady state and time-resolved photoluminescence quenching of streptavidin modified CdSe/ZnS quantum dots (QDs) instigated by biotin-peptide-BHQ-1 (biotin-pep-BHQ-1) molecule was investigated. Here, we have achieved an efficient photoluminescence (PL) quenching of QDs with the conjugation of dark quencher (black hole quencher-BHQ) molecules intermediated with the GPLGVRGK peptide. The luminescence of streptavidin-QDs585 was decreased upon titration with a nano molar concentration of the biotin-GPLGVRGK-BHQ-1 molecule. It has been suggested that the decrease of QDs PL occurred through a Förster resonance energy transfer (FRET) mechanism from the analysis of steady state photoluminescence intensity measurements as well as time resolved lifetime measurements of streptavidin-QDs and QDs-

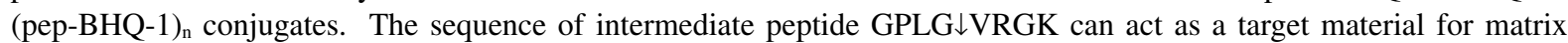
metalloproteinases-2 (MMP-2) produced by cancer cells at its Gly and Val region, shown by the down-headed arrow. Interestingly, here the reported self-assembled QDs-(pep-BHQ-1) ${ }_{n}$ conjugates could detect the presence MMP-2 at a detection limit of $1 \mathrm{ng} / \mathrm{mL}$ with a clear luminescence recovery.
\end{abstract}

Keywords Quantum dot (QD), Förster resonance energy transfer (FRET), black hole quenchers, luminescence quenching, matrix metalloproteinase

(Received January 6, 2017; Accepted January 23, 2017; Published February 10, 2017)

Semiconductor quantum dots, generally $\mathrm{CdSe} / \mathrm{ZnS}$ core/shell QDs are a nano-scale embodiment of one semiconductor inside another semiconductor with a larger bandgap. QDs have recently articulated their performance as an imaging agent in diagnostics as well as bioanalysis. ${ }^{1-4}$ They are bright emitters and can feature narrow emission ranges that are freely tunable across the whole visible spectrum and having long lifetime of the excited states..$^{5-11}$ Moreover, they are widely available, can be made live-cell compatible by organic coatings and standard coupling chemistry, and aside from transitions to metastable dark states, that is fluorescence intermittency or blinking with power-law statistics, they are exceptionally photostable. This means that they can be transferred many times between their ground ("off") and their fluorescent ("on") state. ${ }^{12-14}$ These unique characteristics make them applicable as a useful material in solar cells, ${ }^{15}$ semiconductor LASER, ${ }^{16,17}$ single photon sources, ${ }^{18-20}$ LEDs, $4 \mathrm{~K}$ and $8 \mathrm{~K}$ television displays ${ }^{19}$ and other technologies. QD has recently been used as an imaging agent in single-molecule bioimaging ${ }^{20-22}$ as well as even in STED microscopy. ${ }^{14}$ QD-based western blotting protocols were useful

† To whom correspondence should be addressed.

E-mail: pillai.sreenadh.sasidharan@ @.mbox.nagoya-u.ac.jp for the ultrasensitive detection of "tracer" proteins in cell lysates, ${ }^{23} \mathrm{QD}$-conjugated hybridization probes were effective for a rapid procedure for the selection of highly effective smallinterfering RNA (siRNA) sequences for RNA interference in mammalian cells, ${ }^{24}$ QD-lectin conjugates could identify leukaemia cells from normal lymphocytes using fluorescent confocal microscopy and flow cytometry, ${ }^{25}$ QDs could be transfected into the cytoplasm and even the nucleus of the cells using insect neuropeptide, namely, allatostatin 1 from Drosophila melanogaster. ${ }^{26}$

The conventional luminescence probe only shows monotonous luminescence changes upon biosensing and causes limited resolution. High luminescence quenching efficiency and precision in target selectivity makes the probe ideal for biosensing and high-resolution imaging in complex systems, like living cells. One of the innovative applications of QD is its ability to act as an efficient Förster resonance energy transfer FRET donor material, which is one of the innovative tools for luminescence based biosensing.

MMPs are a family of zinc-dependent proteases, called as matrix metalloproteinase, because of their dependence on metal ions for catalytic activity as well as their potent ability to degrade the structural components of the extra cellular matrix. 
MMP-2 is synthesized as inactive zymogens (proMMPs) and is either secreted into the extra cellular space or anchored to integrin receptors of the cell membrane. The activation of MMP-2 requires removal of the pro-peptide domain by proteolysis to expose the active site with the catalytic domain. MMPs influence diverse physiologic and pathological processes, including aspects of embryonic development, tissue morphogenesis, wound repair, inflammatory diseases, and cancer. ${ }^{27-29}$ A number of MMPs are expressed in various human cancers. MMP-2 is secreted as zymogens usually by stromal cells, such as fibroblasts. ${ }^{30}$ MMP- 2 over expression is strongly linked to melanoma progression and relatively little MMP-2 is observed in normal tissue of the skin. Stem cells are localized in the micro environment known as a stem-cell niche, where they are maintained in undifferentiated and quiescent states. MMPs help the transfer of stem cells from quiescent state to proliferate niches. ${ }^{31}$ Regenerative medicines with the use of stem cells as well as recently iPS cell have appeared as a potential therapeutic alternative for many disease states. The microenvironment modulates the self-renewal, proliferation, and differentiation of stem cells, thus enabling continuous production of all type of mature cells. Proteolysis by MMPs controls the extracellular microenvironment. MMPs are the key factor in regulating the transfer of stem cells from the quiescent niche to the proliferative niche. ${ }^{31-36}$ Hence, it gathered a paramount attention to explore the molecular mechanism of MMPs based proteolysis at the extracellular matrix (ECM).

In this manuscript, we present a simple and reliable method for a molecular sensing technology with on-off luminescence control, using QDs and QD-pep-BHQ-1 conjugates. Here, QDs and BHQ-1 molecule are conjugated using a simple streptavidin-biotin binding method. The intermediated peptide GPLG $\downarrow$ VRGK acts as a bridge between the donor and multiple acceptor molecules, which can be cleaved at the Gly and Val region of the PLGVR amino acid sequence by MMP-2 produced by cancer cells. We further evaluated the sensitivity of this nano assembly system for specific MMP2 enzyme detection.

In the present study, biotin conjugated glycine-proline-leucineglycine-valine-arginine-lysine-BHQ-1- $\mathrm{CONH}_{2}$; biotin-(pepBHQ-1) was chosen as the acceptor and streptavidin functionalized $\mathrm{CdSe} / \mathrm{ZnS}$ core-shell QDs as donor material. Streptavidin functionalized $\mathrm{CdSe} / \mathrm{ZnS}$ QD samples with PL intensity maximum ca. $585 \mathrm{~nm}$ (QD585), $565 \mathrm{~nm}$ (QD565) and $525 \mathrm{~nm}$ (QD525) and $655 \mathrm{~nm}$ (QD655) were purchased from Life Technologies (Tokyo, Japan). The streptavidin-QD585 solution was diluted to $3 \mathrm{nM}$ first with purified water (Millipore Milli-Q Lab purification system, Tokyo, Japan). According to the manufacturer, the QD surface is tethered randomly with streptavidin molecules by making use of carbodiimide (EDC) coupling chemistry. The resultant QD carries 5 - 10 streptavidin molecules, and each streptavidin provides at least 3 sterically available binding sites for biotin binding. In other words, each QD can be conjugated with an average of 15 to 30 biotin-(pepBHQ-1) molecules.

A custom-made peptide, biotin-glycine-proline-leucineglycine-valine-arginine-(lysine BHQ-1)- $\mathrm{CONH}_{2}$ or biotinGPLG $\downarrow$ VRG(Lys[BHQ-1])-CONH ${ }_{2}$, with $99 \%$ purity was purchased from Custom Peptide \& Antibody Service, Eurofins Genomics K. K. (Tokyo, Japan). The downward arrow shows the MMP-2 cleaving site in the PLGVR amino acid sequence. The biotin-pep-BHQ-1 was diluted to $1 \mathrm{mM}$ in DMSO and further diluted to $2 \mu \mathrm{M}$ with purified water (Millipore). Activated MMP-2 was purchased from (Calbiochem, USA); pro-MMP-2 (human MMP-2, Recombinant, Anaspec, USA) and the TCNB buffer $\left(50 \mathrm{mM}\right.$ Tris, $10 \mathrm{mM} \mathrm{CaCl}_{2}, 150 \mathrm{mM} \mathrm{NaCl}$,

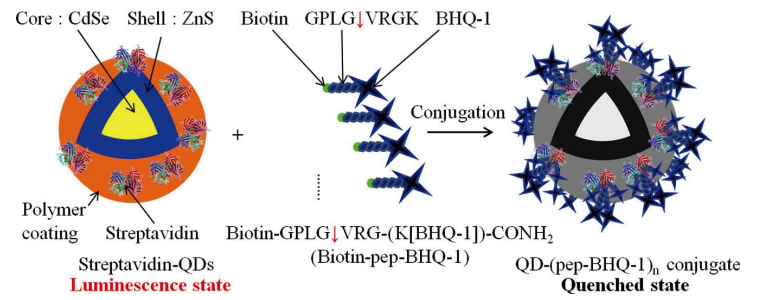

Fig. 1 Schematic diagram of the quenching of quantum dot (QD) photoluminescence by using black hole quencher (BHQ) molecules. This diagram shows that streptavidin-QDs $585 \mathrm{~nm}$ are conjugated with biotin-glycine-proline-leucine-glycine-valine-arginine-(lysine BHQ-1)amide $\left\{\right.$ (biotin-GPLGVRG (K[BHQ-1])-CONH ${ }_{2}$; biotin-pep-BHQ-1) and QD photoluminescence was quenched by BHQ molecules.

and $0.01 \%$ Brij35; pH 7.5) was prepared using $50 \mathrm{mM}$ Tris (Wako), $10 \mathrm{mM} \mathrm{CaCl}_{2}$ (Sigma), $150 \mathrm{mM} \mathrm{NaCl}$ (Sigma), and $0.01 \%$ Brij35 (Thermo Scientific); and the $\mathrm{pH}$ was monitored using a pH meter (Horiba, Japan).

Figure 1 shows a graphical representation of the conjugation procedure. The ratiometric amount of the biotin-pep-BHQ-1 molecule was titrated against a $3 \mathrm{nM}$ QD585 solution. An as-prepared biotin-pep-BHQ-1 sample $(2 \mu \mathrm{M})$ was added in each step of titration against a QD585 (3 nM, $200 \mu \mathrm{L})$. Thus, at each step of titration, it was possible to calculate the apparent QD-pep-BHQ-1 conjugation ratios.

For all titration experiments, purified water (Millipore) was used. The optical cuvette was flushed with acetone and ethanol, cleaned in aqueous $\mathrm{H}_{2} \mathrm{SO}_{4}$, washed with purified water and dried in a nitrogen flow. Steady state PL spectral analysis of the QDs585-pep-BHQ-1 titration experiment was performed by using a fluorescence spectrophotometer F-4500 (Hitachi, Tokyo, Japan); QDs525-pep-BHQ-1, QDs565-pep-BHQ-1 and QDs655-pep-BHQ-1 were performed by using a Fluorolog 3 (Horiba, Tokyo, Japan) respectively. Between the 1-min time gap of successive excitations, titration was performed and the fluorescence intensity was measured. QDs were excited at $400 \mathrm{~nm}$ to maintain the direct excitation of BHQ-1 to be as low as possible. Time resolved fluorescence spectra were recorded using an assembly of a polychromator (250IS, Chromex, Japan) and a photon counting streak-camera (C4334, Hamamatsu, Japan). The QDs585 were selectively excited using $400 \mathrm{~nm}$ pulses (150 fs) generated from the second-harmonic generation (SHG) crystal of an optical parametric amplifier (OPA) (OPA 9400, Coherent Japan). The OPA was pumped by $800 \mathrm{~nm}$ pulses $(200 \mathrm{kHz})$ from a regenerative amplifier (RegA 9000, Coherent) that was seeded by a mode-locked Ti:sapphire laser (Mira 900F, Coherent). The fluorescence signal from the QDs585 was collected through a suitable band-pass filter and focused at the entrance slit of the polychromator.

A unit of QD-(pep-BHQ-1) $n$ conjugated consisted of $585 \mathrm{~nm}$ donor QDs with the 4-10 streptavidin molecule, from the manufacturer's specification (Life Technology), streptavidin may be randomly attached to the QD surface, it seems that this kind of conjugation is made possible by EDC or similar ester coupling chemistry, as described by the manufacturer and presumably result in $2-3$ sterically available binding site per protein; thus, each QD can conjugate with an average of $16-40$ biotin-pep-BHQ-1 molecules. Normalized absorption spectra of the biotin-pepBHQ-1 molecule and the normalized emission intensity of QDs525, QDs565, QDs585, and QDs655 are shown in Fig. 2A. The spectral overlap integral of various QDs donor and biotinpep-BHQ-1 acceptor combination and the corresponding Förster 
$\mathbf{A}$

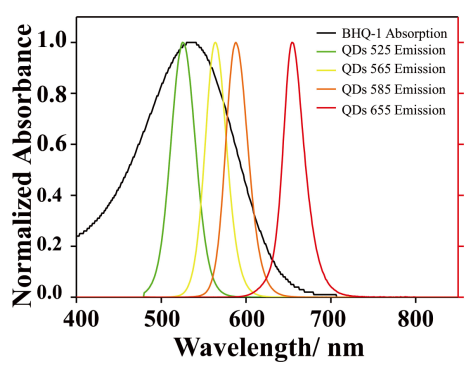

C

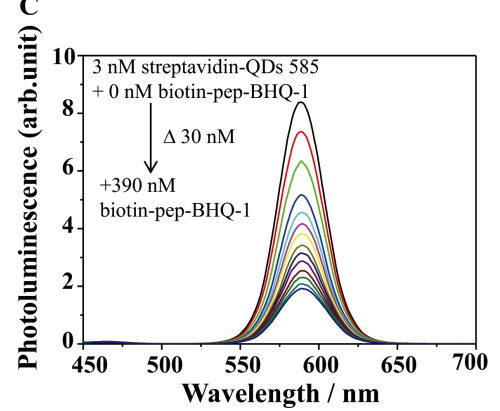

B

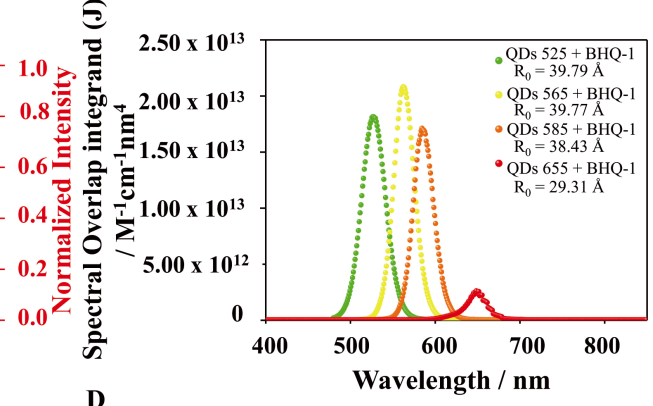

D

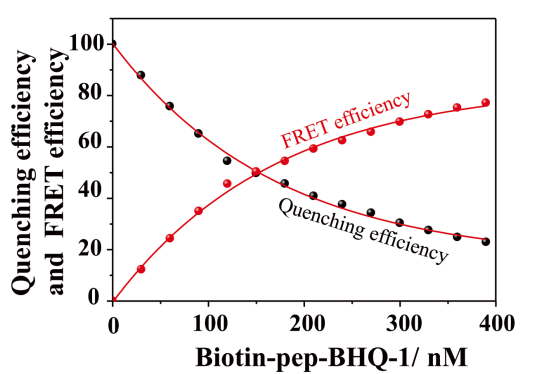

Fig. 2 (A) Normalized absorption spectra of biotin-pep-BHQ-1 molecule and normalized emission intensity of QDs525, QDs565, QDs585 and QDs655. (B) Spectral overlap integral of various QDs donors and biotin-pepBHQ-1 acceptor combinations, the Förster radius $R_{0}$ value for each donor-acceptor combinations has presented. (C) Evolution of the steady-state photoluminescence quenching spectra of QDs585 in the QD-pep-BHQ-1 assemblies; $3 \mathrm{nM}$ streptavidin QDs585 was titrated against various concentrations of the biotin-pep-BHQ-1, $0-390 \mathrm{nM}$ in Milli-Q purified water. Samples were excited at $400 \mathrm{~nm}$. (D) FRET efficiency data from QD-pep-BHQ-1 conjugates. $\mathrm{QD}, \mathrm{PL}$ quenching reported as a percentage in black and the corresponding FRET efficiency as a function of biotinpep-BHQ-1 concentration titrating against $3 \mathrm{nM}$ concentrations of streptavidin QD525 are presented in red.

radius $R_{0}$ value of each donor acceptor are presented in Fig. 2B. It is assumed that the excellent overlap integral between QD585 emission and BHQ-1 absorption allows the biotin-pep-BHQ-1 molecule bound within the streptavidin binding pocket to quench the QD585 nm emission effectively through an energy transfer process. We observed a decreased PL intensities of the QD585 donor in the QD-(pep-BHQ-1) ${ }_{n}$ conjugates compared to the PL intensity of QDs before conjugation to biotin-pep-BHQ-1 as the number of acceptor molecules increases as shown in Fig. 2C. Figure 2D represents the FRET efficiency at various acceptor concentrations. Table S1 gives the calculated values of the FRET efficiencies from the steady state ensemble measurements for QDs585-(pep-BHQ-1)n; Table S2 gives the QDs565-(pep-BHQ-1) n $_{\mathrm{n}}$ conjugates. The quenching efficiency is improved by the presence of $\sim 4-10$ streptavidin, each of which is capable of specifically binding four biotin-pep-BHQ-1 energy acceptors on its tetra pockets, compared to complexes with single donor acceptor FRET pairs. Increasing the number of energy acceptors per QD donor increases the effective acceptor extinction coefficient, which in turn proportionally improves the effective overlap integral for single QD donor-multiple acceptor complexes.

In support of this presumed maximum assembly ratio, the apparent quenching response observed for the biotin-pepBHQ-1 conjugates suggests an underlying bimodal process with an inflection at a ratio of $n=40$ biotin-pep-BHQ-1; the fit for 0 to 40 peptides is quite steep in Fig. S1 while that for subsequent $n \geq 40$ to 130 biotin-pep-BHQ-1 closely resembles the linear quenching by only the BHQ-1 molecule. This would correspond to a highly initial rate of quenching expected for biotin-pep-BHQ-1 directly attached to the QD along with a subsequent much weaker, linear-quenching process driven by Stern-Volmer solution phase collisional quenching interactions.
The Stern-Volmer constant was $1.7 \times 10^{7} \mathrm{M}^{-1}$ based on the slope of a plot up to $n \sim 40$; the apparent bimolecular quenching constant was equal to $1.6 \times 10^{15} \mathrm{M}^{-1} \mathrm{~s}^{-1}$. The various reasons for a deviation from the theoretical value of streptavidin-biotin binding constant has been described extensively in previous studies. $^{37}$ It should be considered that the solid support for streptavidin-biotin interaction significantly affects the kinetics of the reaction due to the diffusion limitation and steric hindrance. ${ }^{38,39}$ The steady state luminescence time evolution spectra of QD PL quenching upon titration with different concentration of biotin-pep-BHQ-1 was performed as shown in Fig. S3; it is observed that as the concentration of biotin in solution increases the streptavidin-biotin binding rate also increases. Also, there is no evidence of any cooperative interaction between the biotin molecules on the surface of same particle with streptavidin on its surface. ${ }^{39}$ Hence, the lower concentration of biotin upon stoichiometric titration, QDs solid surface induced diffusion limitation and steric hindrance reduces the streptavidin-biotin binding reaction kinetic rates and shows deviation from the theoretically predicted value of the binding constant.

In addition to steady state analysis, Fig. 3A shows the timeresolved excited state lifetime analysis of QD-(pep-BHQ-1)n conjugates at various acceptor concentrations. The timeresolved PL of the semiconductor quantum dot directly reflects the transient population that is distributed in the radiative core states. The multiexponential dynamics of the excitonic decay of colloidal CdSe QDs are due to the heterogeneity in size, shape, degree of aggregation, surface passivation and structure within a given quantum dot sample. ${ }^{40-42}$ This heterogeneity leads to a variety of decay processes available to band-edge excitons, where each observed time constant, $\tau$, is an average excited state lifetime for a population of QDs with a set of available decay 

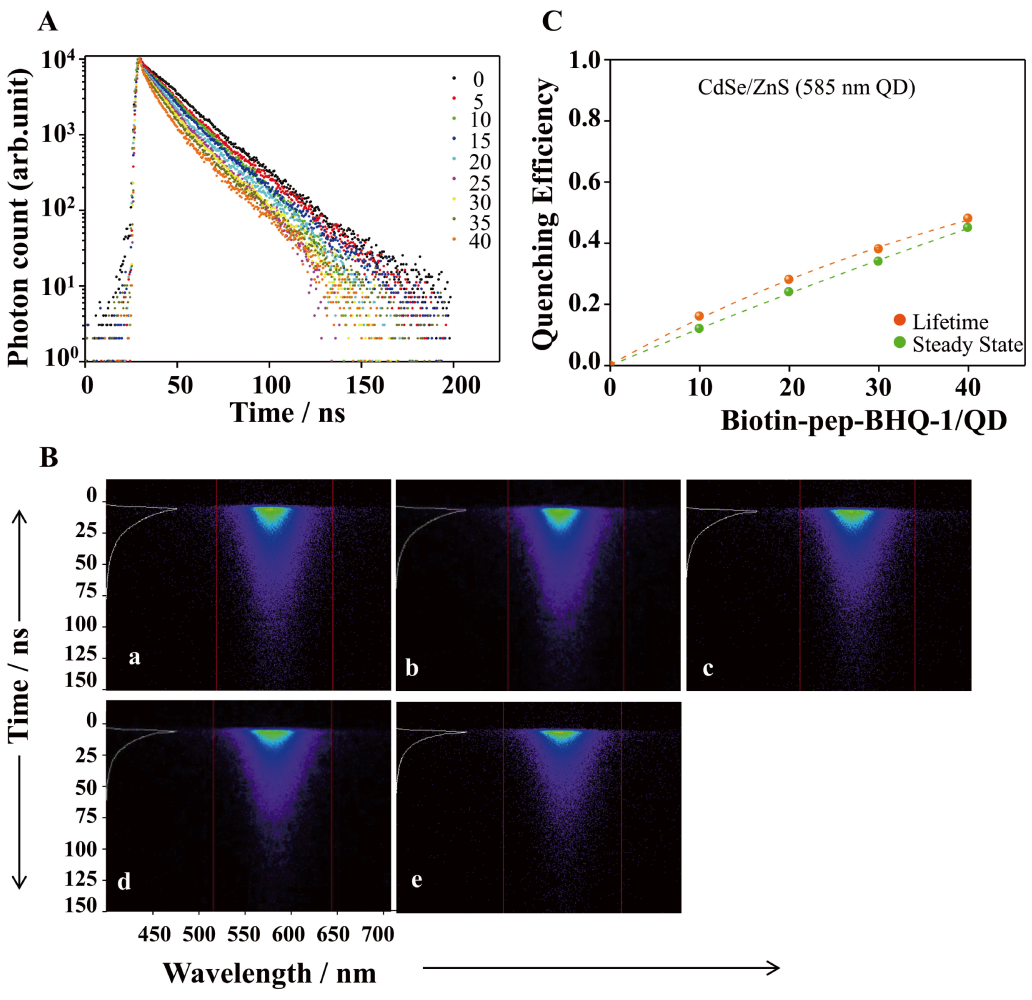

\begin{abstract}
Fig. 3 (A) Time-resolved excited state lifetime analysis from select QD-(pep-BHQ-1) conjugates. Streptavidin QD585 conjugated with the indicated increasing ratios of biotin-pep-BHQ-1. QD are excited at low absorption wavelength of BHQ-1 molecule at $400 \mathrm{~nm}$ using $150 \mathrm{fs}$ laser pulses $\left(6.3 \mathrm{~W} / \mathrm{cm}^{2}\right)$. (B)

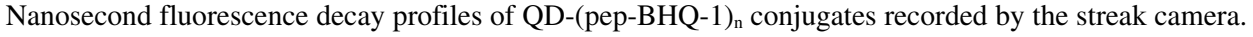
(C) Comparison of normalized quenching profiles from steady-state versus time resolved PL decay data of QDs585 nm versus increasing ratio of biotin-pep-BHQ-1 assembled per QD. Normalized steady state data are plotted in green while the corresponding time-resolved PL decay is shown in orange.
\end{abstract}

pathways ranging in time scale from hundreds of femptosecond to microseconds. ${ }^{43-49}$

In addition to radiative recombination, decay of the excited state population depends on several competing radiationless processes such as Auger relaxation of hot electrons, ${ }^{47,50}$ spin relaxation, ${ }^{51-53}$ phonon induced carrier relaxation, ${ }^{54}$ biexciton decay, ${ }^{55}$ carrier traping ${ }^{56,57}$ at nanocrystal defects, charge transfer into ligand based orbital and relaxation to the ground state. For the convenience of researchers, second or third-order non-linear fitting is applied from time to time to in order to fit the decay profile. Here, we have used a third-order exponential fit to iterate the average lifetime of the QDs and QD-(pep-BHQ-1)n conjugates.

In the present case, the two decay constants shall be assigned to the Auger process and a combination of spin-allowed and forbidden relaxations when there is no energy transfer. Indeed, FRET or any other fast relaxation process suppresses the intrinsic slow radiative recombination processes. As a result, the fast process becomes clear during energy transfer, because the energy transfer competes with the relatively slow relaxations in the dark exciton state or surface states. The FRET efficiency of QD585-(pep-BHQ-1) n $_{\text {n }}$ conjugates at various numbers of acceptors are calculated using Eq. (6) (Supporting Information). The estimated FRET efficiency values are given in the Table S1. Figure 3B shows streak-camera images of the nanosecond PL decay profiles for QD585-(pep-BHQ-1) ${ }_{n}$ conjugates at different acceptor concentrations. Consistent with the steady state results, the average exciton lifetime $\left(\tau_{\mathrm{av}}\right)$ decreases with increasing biotin-pep-BHQ-1 assembled per QD. A comparison of the QD quenching efficiency derived from steady state and time- resolved PL data is shown in Fig. 3C. The steady-state quenching efficiency is slightly lower than the lifetime decay at lower acceptor concentrations and become comparable as the concentration increases.

The concentric array of increasing numbers of biotin-pepBHQ-1 around each QD makes FRET appreciable in this assembly. Arraying an increasing number of acceptors around the QD eventually causes a proportional increase in the FRET acceptor extinction coefficient through an increased absorption cross-section. This also increases the probability of FRET occurrence between the QD donors to one of the BHQ-1 acceptors.

Previous studies showed that FRET rate between the QD donors and acceptors was wavelength dependent, within the same population and under the same condition are prone to undergo very different FRET quenching efficiency due to differences in their spectral overlap. ${ }^{58}$ The ensemble QD PL profile is made up of continuum of slightly different type quantum dots that emit at slightly different wavelength with individual PL emission line widths that are significantly narrower than the overall ensemble spectrum.

The presence of wavelength-dependent PL quenching in QD donor-acceptor system serves to provide strong evidence of an underlying FRET process, while its absence can suggest the presence of other quenching mechanisms. To confirm the wavelength dependency of the PL quenching process, various wavelength emitting QDs with a constant number biotin-pepBHQ-1 acceptors were conjugated by keeping a molar ratio of $1: 40$, the steady state spectral profiles and resulting quenching efficiency are presented in Fig. S2. In particular, the 
$\mathbf{A}$

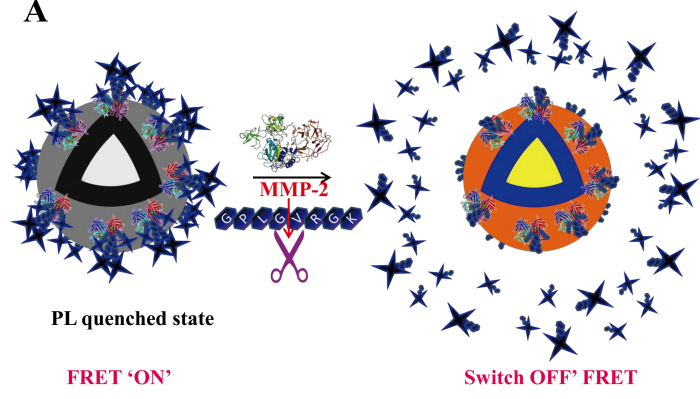

B

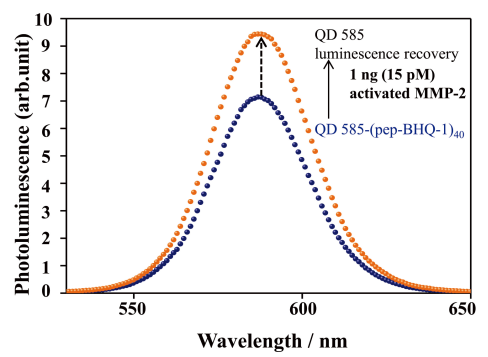

Fig. 4 (A) Hypothesis about FRET-mediated PL quenching of QDs, followed by sensitive detection of MMP-2 using QD-(pep-BHQ-1)n conjugates. (B) Detection of MMP-2 with QD-(pep-BHQ-1) conjugates. The QD-(pep-BHQ-1 $)_{40}$ conjugates were incubated with activated MMP-2 $(1 \mathrm{ng} / \mathrm{mL})$ for $2 \mathrm{~h}$ at $37^{\circ} \mathrm{C}$ before FRET measurement. From bottom to top, the black line shows the PL quenched state and the red line shows the recovered PL spectra of QD585 after $2 \mathrm{~h}$ of incubation with $1 \mathrm{ng} / \mathrm{mL}$ of activated MMP-2.

stoichiometric control achieved here allowed us to examine the system at different state of efficiencies, which in other way help us for the quantitative evaluation of target sites and thus target specificity of biomolecules in a centrosymmetrically arranged QD protein sensor.

The activated MMP-2 was incubated with a QD-pep-BHQ-1 sample at $37^{\circ} \mathrm{C}$ before the FRET measurement. The FRET ratio for the control was also checked for a prolonged time at $37^{\circ} \mathrm{C}$ without the presence of MMP2 and with the presence of a proMMP2 sample. The measurements were performed between a 4-min time gap for the former and a 10-min time gap for the later under $400 \mathrm{~nm}$ excitation, for $\geq 1 \mathrm{~h}$. It was observed that the system remained unchanged and continued its PL quenched state during the entire measurement as shown in Figs. S4 and S5. We performed the experiment at a MMP2 concentration of $1 \mathrm{ng} / \mathrm{mL}$ (15 pM), which was a higher sensitivity compared to the limit of detection for the previous MMP-2 detection experiments. ${ }^{59-61}$ A previously reported QD-based BRET sensor detected MMPs at a detection limit of $2 \mathrm{ng} / \mathrm{mL}$ with a $10 \%$ decrease in the BRET ratio, ${ }^{59}$ but a QD based FRET sensor detected at a detection limit of $0.5 \mu \mathrm{g} / \mathrm{mL}^{60}$ and a magnetic sensor detected at a detection limit of $19 \mathrm{ng} / \mathrm{mL}^{61}$ which are comparatively at a lower sensitivity than reporter here with QD-

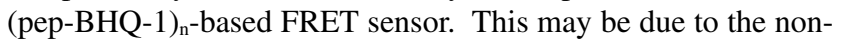
fluorescent property of a black hole quencher acceptor molecule with high a luminescence quenching ability. MMP2 $(1 \mathrm{ng} / \mathrm{mL})$ was incubated with the QD585-(pep-BHQ-1) 40 assembly in TCNB buffer $(50 \mathrm{mM}$ Tris, $10 \mathrm{mM} \mathrm{CaCl}, 150 \mathrm{mM} \mathrm{NaCl}$, and $0.01 \%$ Brij35; pH7.5) at $37^{\circ} \mathrm{C}$ for $2 \mathrm{~h}$. It was observed that there was a $20 \%$ PL recovery of QD585 compared to the PL quenched state as presented in Fig. 4B.

In summary, streptavidin QDs with different wavelength were conjugated to black hole quencher (BHQ-1) molecules intermediated with the MMP-2 sensing target peptide. The PL quenching of QDs in the QDs-(pep-BHQ-1)n conjugates was studied using steady-state and time resolved ensemble PL measurements. Here, we contribute more to the understanding of QD PL quenching through the wavelength-dependent PL quenching process between streptavidin-QDs (donor) and biotin-pep-BHQ-1 (acceptor) by steady state and time-resolved photoluminescence analysis. The sequence of the intermediate peptide was GPLG $\downarrow$ VRGK, which can be catalytically cleaved by MMPs produced by cancer cells at the Gly-Val region. Here the reported QD-pep-BHQ-1 nanoassembly could detect the presence of MMP-2 at a $1 \mathrm{ng} / \mathrm{mL}$ concentration. MMP-2 proteases usually attach at the integrin receptors of the cell membrane; future experiments will check the MMP-2 detection ability of QDs585-pep-BHQ-1 at the extra cellular environment (ECM) of cancer cells. Advanced functional QD-FRET based conjugates have been developed for a variety of biosensing applications, it is predicted that nanomaterials-based biosensor will be helpful for future enzyme sensitive regenerative medicine and theranostics application. ${ }^{62,63}$ It is clear from this study that a controlled orientation and a bioconjugation architecture are needed for a highly specific and functional oriented development of a future energy transfer based nanobiosensor. Sophisticated techniques are needed to unravel the complex structure-function relationship of these hetero nano assembled complex photonic system for bioanalysis.

\section{Acknowledgements}

This research is supported by the Japan Agency for Medical Research and Development (AMED) through its "Research Center Network for Realization of Regenerative Medicine". V. B. thank MEXT for a JSPS Grant-in-Aid for scientific research on innovative areas "Photosynergetics" (KAKENHI Grant 15H01099). This work was partially supported by JSPS KAKENHI Grant Number JP26790006.

\section{Supporting Information}

Supplementary information has been attached along with the manuscript. This material is available free of charge on the Web at http://www.jsac.or.jp/analsci/.

\section{References}

1. R. Bakalova, H. Ohba, Z. Zhelev, M. Ishikawa, and Y. Baba, Nat. Biotechnol., 2004, 22, 1360.

2. R. Bakalova, H. Ohba, Z. Zhelev, T. Nagase, R. Jose, M. Ishikawa, and Y. Baba, Nano Lett., 2004, 4, 1567.

3. X. Wu, H. Liu, J. Liu, K. N. Haley, J. A. Treadway, J. P. Larson, N. Ge, F. Peale, and M. P. Bruchez, Nat. Biotechnol., 2002, 21, 41 .

4. R. Gill, M. Zayats, and I. Willner, Angew. Chem. Int. Ed., 2008, 47, 7602 .

5. V. Biju, Y. Makita, A. Sonoda, H. Yokoyama, Y. Baba, and M. Ishikawa, J. Phys. Chem. B, 2005, 109, 13899.

6. V. Biju, Y. Makita, T. Nagase, Y. Yamaoka, H. Yokoyama, Y. Baba, and M. Ishikawa, J. Phys. Chem. B, 2005, 109, 14350.

7. R. Jose, N. U. Zhanpeisov, H. Fukumura, Y. Baba, and M. Ishikawa, J. Am. Chem. Soc., 2006, 128, 629.

8. R. Jose, Z. Zhelev, R. Bakalova, Y. Baba, and M. Ishikawa, Appl. Phys. Lett., 2006, 89, 13115.

9. Y.-S. Park, A. Dmytruk, I. Dmitruk, A. Kasuya, Y. Okamoto, N. Kaji, M. Tokeshi, and Y. Baba, J. Phys. Chem. C, 2010 , $114,18834$.

10. Z. Zhelev, R. Bakalova, H. Ohba, R. Jose, Y. Imai, and 
Y. Baba, Anal. Chem., 2006, 78, 321.

11. Y. S. Park, A. Dmytruk, I. Dmitruk, A. Kasuya, M. Takeda, N. Ohuchi, Y. Okamoto, N. Kaji, M. Tokeshi, and Y. Baba, ACS Nano, 2010, 4, 121

12. V. Biju, R. Kanemoto, Y. Matsumoto, S. Ishii, S. Nakanishi, T. Itoh, Y. Baba, and M. Ishikawa, J. Phys. Chem. C, 2007, 111,7924

13. V. Biju, Chem. Soc. Rev., 2014, 43, 744.

14. J. Hanne, H. J. Falk, F. Görlitz, P. Hoyer, J. Engelhardt, S. J. Sahl, and S. W. Hell, Nat. Commun., 2015, 6, 7127.

15. P. V. Kamat, J. Phys. Chem. Lett., 2013, 4, 908.

16. V. I. Klimov, A. A. Mikhailovsky, S. Xu, A. Malko, J. A. Hollingsworth, C. A. Leatherdale, H. Eisler, and M. G. Bawendi, Science, 2000, 290, 314

17. V. I. Klimov, S. A. Ivanov, J. Nanda, M. Achermann, I. Bezel, J. A. McGuire, and A. Piryatinski, Nature, 2007, 447, 441.

18. A. Imamoğlu, P. Michler, M. D. Mason, P. J. Carson, G. F. Strouse, and S. K. Buratto, Nature, 2000, 406, 968.

19. K. Bourzac, Nature, 2013, 493, 283.

20. M. Sugawa, S. Nishikawa, A. H. Iwane, V. Biju, and T. Yanagida, Small, 2010, 6, 346.

21. N. Kawashima, K. Nakayama, K. Itoh, T. Itoh, M. Ishikawa, and V. Biju, Chem.-Eur. J., 2010, 16, 1186.

22. N. Kaji, M. Tokeshi, and Y. Baba, Chem. Rec., 2007, 7, 295.

23. R. Bakalova, Z. Zhelev, H. Ohba, and Y. Baba, J. Am. Chem. Soc., 2005, 127, 9328.

24. R. Bakalova, Z. Zhelev, H. Ohba, and Y. Baba, J. Am. Chem. Soc., 2005, 127, 11328

25. Z. Zhelev, H. Ohba, R. Bakalova, R. Jose, S. Fukuoka, T. Nagase, M. Ishikawa, and Y. Baba, Chem. Commun., 2005, 13,1980 .

26. V. Biju, D. Muraleedharan, K. Nakayama, Y. Shinohara, T. Itoh, Y. Baba, and M. Ishikawa, Langmuir, 2007, 23, 10254.

27. M. D. Sternlicht and Z. Werb, Annu. Rev. Cell Dev. Biol., 2001, 17, 463.

28. K. Kessenbrock, V. Plaks, and Z. Werb, Cell, 2010, 141, 52.

29. M. Egeblad and Z. Werb, Nat. Rev. Cancer, 2002, 2, 161.

30. R. Kalluri and M. Zeisberg, Nat. Rev. Cancer, 2006, 6, 392.

31. V. Plaks, N. Kong, and Z. Werb, Cell Stem Cell, 2015, 16, 225.

32. Z. Werb, Cell, 1997, 91, 439.

33. B. Heissig, K. Hattori, S. Dias, M. Friedrich, B. Ferris, N. R. Hackett, R. G. Crystal, P. Besmer, D. Lyden, M. A. S. Moore, Z. Werb, and S. Rafii, Cell, 2002, 109, 625.

34. K. Kessenbrock, C.-Y. Wang, and Z. Werb, Matrix Biol., 2015, 44, 184.

35. H. M. Blau, T. R. Brazelton, and J. M. Weimann, Cell, 2001, 105, 829.

36. L. Li and W. B. Neaves, Cancer Res., 2006, 66, 4553.

37. S. C. Huang, M. D. Stump, R. Weiss, and K. D. Caldwell, Anal. Biochem., 1996, 237, 115.

38. S. C. Huang, H. Swerdlow, and K. D. Caldwell, Anal.
Biochem., 1994, 222, 441.

39. E. V. Piletska and S. A. Piletsky, Langmuir, 2010, 26, 3783.

40. M. G. Bawendi, W. L. Wilson, L. Rothberg, P. J. Carroll, T. M. Jedju, M. L. Steigerwald, and L. E. Brus, Phys. Rev. Lett., 1990, 65, 1623.

41. A. P. Alivisatos, T. D. Harris, P. J. Carroll, M. L. Steigerwald, and L. E. Brus, J. Chem. Phys., 1989, 90, 3463.

42. D. J. Norris, M. Nirmal, C. B. Murray, A. Sacra, and M. G. Bawendi, Z. Phys. D, Atom. Mol. Cl., 1993, 26, 355.

43. D. M. Mittleman, R. W. Schoenlein, J. J. Shiang, V. L. Colvin, A. P. Alivisatos, and C. V. Shank, Phys. Rev. B, 1994, 49, 14435.

44. H. Wang, C. De Mello Donegá, A. Meijerink, and M. Glasbeek, J. Phys. Chem. B, 2006, 110, 733 .

45. S. Sewall, R. Cooney, K. Anderson, E. Dias, and P. Kambhampati, Phys. Rev. B, 2006, 74, 1.

46. P. Kambhampati, Acc. Chem. Res., 2011, 44, 1.

47. V. I. Klimov, D. W. McBranch, C. A. Leatherdale, and M. G. Bawendi, Phys. Rev. B, 1999, 60, 13740.

48. M. Jones and G. D. Scholes, J. Mater. Chem., 2010, 20, 3533.

49. K. E. Knowles, E. A. McArthur, and E. A. Weiss, ACS Nano, 2011, 5, 2026.

50. S. Xu, A. A. Mikhailovsky, J. A. Hollingsworth, and V. I. Klimov, Phys. Rev. B, 2002, 65, 45319.

51. F. Masia, N. Accanto, W. Langbein, and P. Borri, Phys. Rev. Lett., 2012, 108, 87401.

52. M. J. Fernée, Y. Louyer, P. Tamarat, and B. Lounis, Phys. Rev. Lett., 2012, 109, 229701.

53. L. Biadala, Y. Louyer, P. Tamarat, and B. Lounis, Phys. Rev. Lett., 2009, 103, 37404.

54. P. Guyot-Sionnest, B. Wehrenberg, and D. Yu, J. Chem. Phys., 2005, 123, 74709.

55. S. L. Sewall, R. R. Cooney, K. E. H. Anderson, E. A. Dias, D. M. Sagar, and P. Kambhampati, J. Chem. Phys., 2008, 129,84701

56. M. Jones, S. S. Lo, and G. D. Scholes, Proc. Natl. Acad. Sci. U. S. A., 2009, 106, 3011.

57. C. de Mello Donegá, M. Bode, and A. Meijerink, Phys. Rev. B, 2006, 74, 85320 .

58. T. Pons, I. L. Medintz, M. Sykora, and H. Mattoussi, Phys. Rev. B, 2006, 73, 245302

59. H. Yao, Y. Zhang, F. Xiao, Z. Xia, and J. Rao, Angew. Chem. Int. Ed., 2007, 46, 4346.

60. L. Shi, V. De Paoli, N. Rosenzweig, and Z. Rosenzweig, J. Am. Chem. Soc., 2006, 128, 10378.

61. M. Zhao, L. Josephson, Y. Tang, and R. Weissleder, Angew. Chem., 2003, 115, 1413.

62. P. D. Howes, R. Chandrawati, and M. M. Stevens, Science, 2014, 346, 1247390.

63. B. J. Johnson, W. R. Algar, A. P. Malanoski, M. G. Ancona, and I. L. Medintz, Nano Today, 2014, 9, 102. 